REVISTA ANDALUZA DE ANTROPOLOGÍA.

NÚMERO 9: LA REPRESENTACIÓN DE LAS CULTURAS EN LA MUSEOLOGÍA ANTROPOLÓGICA DEL ESTADO ESPAÑOL

SEPTIEMBRE DE 2015

ISSN 2174-6796

[pp. 222 -231]

http://dx.doi.org/10.12795/RAA.2015.i09.11

\title{
AIX GRACIA, FRANCISCO (2014) Flamenco y poder. Un estudio desde la sociología del arte. Madrid: Fundación SGAE, 555 pp.
}

David Florido

Alicia Reigada

Departamento de Antropología Social

Universidad de Sevilla

Introducción: planteamiento de partida

Nos encontramos ante un sólido trabajo de análisis cultural ya reconocido con el Premio a la Mejor Tesis Doctoral de la Fundación Autor y con el segundo premio de Investigación Cultural Marqués de Lozoya (2013), que tiene por objeto definir el flamenco como campo de arte. Al decantarse el autor por este planteamiento, se inscribe de lleno en un debate que lleva madurando en los ámbitos de la sociología y antropología dedicada al flamenco desde mediados de los noventa. A saber, la consideración del flamenco, bien como una expresión cultural -perspectiva teórica preferida por algunos antropólogos como Cristina Cruces (1996, 2002, 2005), Antonio Mandly (2010), autor no mencionado en el texto, o Isidoro Moreno (1996)- , bien como un género artístico -enfoque que inauguró Gerard Steingress (1993) a partir de la revolucionaria obra de Luis Lavaur sobre la vinculación entre el flamenco y el romanticismo (1976). Francisco Aix, a partir 
del concepto de campo de producción artística de Pierre Bourdieu (1995), se abona a la interpretación de Steingress, su director de tesis, que se acerca al flamenco como género artístico.

Este debate atañe a cuestiones básicas para la comprensión del flamenco como fenómeno socio-cultural. A saber, la ubicación cronológica del flamenco, la identificación de sus protagonistas (qué actores lo producen y lo reproducen históricamente), qué funciones sociales cumple, qué tejidos simbólicos es capaz de trazar, o cómo definir su dinámica histórica. Como el propio autor reconoce en la introducción, la discusión tiene como trasunto otra más profunda, que afecta a la consideración de la cultura: si es dominante en la antropología definirla como elemento constituyente de la sociedad, Aix prefiere comprenderla como un ámbito específico de producción simbólica mediante un código, en este caso artístico, lo que obliga a definir los sectores sociales que producen, transmiten y usan de ese lenguaje. El autor prefiere los términos de "dimensiones" -la etnicitaria y la artística- y destaca que unas y otras lecturas han estado estando presente a lo largo de la historia de las ideas sobre el flamenco: Demófilo (1881), García Lorca/Manuel de Falla (1922) o el tándem Mairena/Molina (1965).

La consideración del flamenco como arte y de éste como un código cultural específico obliga al analista a pensar qué capacidad tienen los actores sociales de ir configurando ese campo de acción simbólica. Como también nos obliga a plantearnos si ese campo es una mera plasmación de valores y modos de expresión del marco social global en el se inscribe (de modo que habría una relación especular entre sociedad y cultura), o si, por el contrario, el flamenco coadyuva en la constitución de la sociedad en la que subsiste. En la trastienda de estas cuestiones subyacen preguntas de calado acerca de si el flamenco es una manifestación popular tradicional o, si por el contrario, fue resultado de la "invención"1 de artistas, intelectuales o/y empresarios, a partir de determinados elementos de una cultura popular (quiere decirse, de clases trabajadoras, minorías étnicas, algunos grupos profesionales) que estaba en un profundo proceso de transformación/disolución durante el siglo XIX. Este debate, además, puede conducirnos a cuestiones como la autoría dentro del campo artístico (la tradición versus los artistas), cuáles han sido los contextos de génesis y desarrollo (urbanos y comerciales versus rurales, profesionales, subalternos), o a

1. Es fundamental tener en cuenta la etimología de esta palabra, que nos permite recuperar su sentido de "hacer venir, hacer traer" [invenire]; esto es, nos indica que los actores sociales, en un momento histórico determinado, usan elementos de la tradición cultural a modo de retales para configurar un tejido cultural nuevo (artístico en este caso). Se puede apreciar con claridad que las funciones y los significados del nuevo artefacto cultural difieren del de aquellos elementos que han sido invocados. En el caso del flamenco, si queremos aplicarle el concepto de "invención de la tradición", hablaríamos de un lenguaje musical y dancístico que se va constituyendo en el siglo XIX por profesionales y que haría uso de elementos poéticos, musicales y dancísticos de distintas formas de cultura popular tradicional, en un contexto histórico en el que esas formas están en proceso de desintegración, junto con las formas sociales que las acompañan. Es en este sentido en el que, a nuestro entender, se expresan tanto Luis Lavaur como Gerard Steingress. 
cuestionarnos sobre los procesos de producción y reproducción (convención y tradición versus innovación y originalidad). Del mismo modo, nos parece un acierto indudable el haber introducido como eje de análisis las formas de recepción del arte flamenco, pues los aficionados y el público en general se incorporan a la pugna por la definición que dé legitimidad al producto flamenco, afectando por tanto a ese proceso de producción y reproducción social. En definitiva, el principal aporte del libro es situar el análisis del flamenco en un debate tan fructífero, al hilo del cual han de responderse cuestiones tan relevantes.

El planteamiento de Aix, que queda justificado en el apartado 1.2, es impecable, siempre y cuando no anule la posibilidad de que el flamenco también es abordable a partir de su otra dimensión, que el autor la denomina representacional, tradicionalista, o etnicitaria. Es decir, desde su punto de vista, fruto de un discurso mixtificador. Lo que no compartimos con Aix es precisamente que no reconozca la posibilidad de una estrategia investigadora sobre el flamenco que lo estudie injerto en códigos y reglas culturales de determinados colectivos sociales, estrategia que estaría orientada a un objeto diferente de investigación -por ejemplo: formas de aprendizaje, sagas familiares, formas expresivas en contextos cotidianos, etc.- y mediante el uso intensivo de técnicas etnográficas. Esta reluctancia se puede explicar tanto por el hecho cierto de que estas reglas y códigos hayan sobrepasado su espacio social original, influyendo en el campo artístico, como por el hecho de que la literatura antropológica que se ha dedicado a ello le parezca a Francisco Aix insuficiente, atrapada en una visión "nacionalista, regionalista o patriotera" (p. 23).

$Y$ es que la contraposición entre la lectura del flamenco como campo de producción cultural (un género artístico) y como expresión cultural, además, trae engarzadas otras cuestiones de fondo, como la identidad étnico-nacional de una manifestación que se internacionaliza prácticamente desde el mismo momento en que emerge como género artístico (mediados del siglo XIX). Esta última cuestión es un debate que traspasa la discusión académica para alcanzar el plano político, y este es un tema específicamente abordado: en qué medida este arte ha sido usado históricamente como "proveedor etnicitario" por el poder gubernamental, en cada momento con objetivos y circunstancias específicas. A lo largo del siglo XIX, el flamenco jugó su papel en la consolidación del proyecto nacional del Estado español (Steingress, 1996), al suministrar una inventada (en el sentido apuntado) cultura popular como representación colectiva que sirviese de vertebración social. Durante el régimen franquista, el denominado "nacionalflamenquismo" continuaba el nacionalismo español anterior, al tiempo que situaba a España en el mercado de bienes simbólicos convertibles en capital económico gracias al turismo incipiente. $\mathrm{Y}$ a lo largo del gobierno autonómico ha devenido en referente cultural para dar solidez a la heterogénea trama cultural de Andalucía y dotarla de un discurso etnicitario reconocible internacionalmente. $\mathrm{Y}$ es que la mercantilización ha 
sido otra posibilidad de instrumentalización, presente en el flamenco desde su propio origen como género artístico. Este asunto es trascendental para los autores que defienden la visión artística del flamenco, pues, con razón, subrayan que el flamenco artístico es moderno, urbano y mercantil, por mucho que use como fuentes culturales (en algunos de sus elementos expresivos) retazos de la cultura popular subalterna arraigados en otros modos de producción social, premodernos.

\section{Marco teórico}

El capítulo segundo fundamenta el marco teórico de partida, la sociología del arte de Arnold Hauser y de Pierre Bourdieu, el cual le permite alejarse de la concepción idealista y romántica del arte y situarlo, por el contrario, en el curso de la historia y de las determinaciones económicas, políticas y sociales que configuran el arte (flamenco en este caso) como campo de producción cultural. Esta perspectiva le llevará a revisar, entre otros aspectos, uno de los pilares fundamentales del sistema moderno de arte, el mito del artista como genio creador y libre. A partir de las enseñanzas de Hauser al respecto, Aix se detiene en el concepto de originalidad como elemento constitutivo del arte. Si bien compartimos con el autor el interés que suscita esta cuestión para comprender la dimensión artística en el flamenco -y en otras artes, tal y como ha apuntado Lourdes Méndez (2009) para el caso de las artes plásticas-, los factores relativos a los requerimientos del mercado podrían complementarse con otro tipo de factores (intelectuales, socioculturales, institucionales) que emergen de la genealogía de conceptos próximos al de "originalidad", como la noción de "creatividad" - la tarea asumida en este sentido por Wladyslaw Tatarkiewicz (1988), en su intento por tender puentes entre la Historia de la Filosofía y la Historia del Arte, resultaría de gran interés al respecto. Por otra parte, en Pierre Bourdieu hallará las claves para aplicar al caso del flamenco el análisis de la distinción, que sirve para formular una economía de los bienes simbólicos, y la teoría de los campos de producción cultural con sus conceptos asociados -autonomía relativa, agencia, habitus, capital cultural, legitimidad.

Otra de las cuestiones teóricas que atraviesan el libro es la de las lecturas patrimonialistas del flamenco. Aquí el autor toma indistintamente en consideración tanto la versión académica (a lo Cristina Cruces, 2001), como la social (que es expresada en ruedas de prensa, en escritos divulgativos y, en general, en las manifestaciones al uso sobre el flamenco en el juego social). Acertadamente, el autor llama la atención sobre los efectos que las iniciativas patrimonializadoras institucionales puedan tener sobre el propio bien protegido/incentivado (el arte flamenco, en este caso), en cuanto a su valor simbólico, su recepción por el público, su dinamicidad... Se trata, de hecho, de una de las cuestiones que pueden generar un debate más contradictorio y discutible tanto en el ámbito académico como en el social. 
Compartimos con el autor su idea de que el patrimonio, más que un elemento sustantivo constituido por atributos culturales que quedan asociados a un determinado referente (visión esencialista e irremediablemente reificadora), es un proceso resultado de la pugna social entre distintas "agencialidades". Así, el patrimonio no descansa tanto en las cualidades y características objetivas, sino más bien en el proceso de negociación/ conflicto, de carácter político, mediante el cual se inicia la patrimonialización. Esta interpretación del concepto, ya asentada en la literatura especializada (Ariño, 2002), no es la que ha permeado los discursos sociales (de políticos, agentes publicitarios, críticos, artistas, gente común), por lo tanto sería preceptivo delimitar el concepto académico del social que el autor usa indistintamente.

La trama conceptual relativa al patrimonio termina por complicarse aún más en el apartado dedicado a los usos del patrimonio (2.5), usando ahora la categoría con una acepción diferente a las anteriores. En este caso, Aix hace referencia a los usos instrumentales de la herencia artística dentro del campo. De este modo, establece una fructífera diferenciación entre "patrimonialidades" - estrategias desde agencias artísticas situadas en posiciones de poder para apropiarse de determinados capitales culturales del arte: una tendencia, una escuela...- y "usos del patrimonio" -para referirse a tácticas de infrapolítica de los distintos actores para situarse en el campo artístico desde una posición subordinada, las más de las veces tildada de herética por las patrimonialidades dominantes-. Un acierto del autor es aplicar estas categorías no sólo a las visiones tradicionalistas -las que normalmente pretenden acotar el espacio estético de la "pureza"-, sino a las propuestas emergentes e innovadoras, que reivindican la autonomía del artista respecto de cualquier hipoteca tradicionalista etnicitaria.

Aix reconoce la importancia de un análisis genealógico del proceso de producción del campo artístico del flamenco, subrayando tres hitos: (i) el tránsito del siglo XIX al XX (período no abordado en esta obra); (ii) el momento de reacción purista entre los años 50 y 70 del s. XX, conocido en la jerga académica como "neojondismo", que significó "su estatuto de autonomía artística" (p. 45) y que se fundamentó intelectualmente en el mairenismo (período al que queda dedicado el capítulo tercero); y (iii) el tránsito del siglo XX al XXI, acuñado como flamenco "posmoderno" por sus defensores, contrapuesto al anterior por estar soportado por discursos y prácticas rupturistas pero muy condicionado por los intentos de instrumentalización política por parte de la Administración Autonómica (período al que se dedica el cuarto capítulo).

\section{Aplicación del marco teórico a la historia reciente del flamenco}

Es sugerente la aplicación que hace el autor de estos conceptos a procesos históricos concretos: el capítulo tercero desarrolla la transformación del mairenismo de una propuesta gitanista (hermetismo) enmarcable en la categoría táctica/uso del patrimonio hasta convertirse en un discurso intelectual legitimador, un ejemplo de patrimonialidad 
estratégica que consigue un locus de poder y reconocimiento desde el cual pontificar la estética del flamenco. Del mismo modo que puede resultar esclarecedor su afirmación de que este proceso de revalorización social del flamenco tuvo un marcado carácter político, que se fue trasfigurando de una primera versión conservadora (Molina, Pemán, González Climent) a otra rupturista, y de izquierdas, respecto al régimen franquista (Moreno Galván y Meneses, Morente, entre otros). Este movimiento, si bien consiguió la institucionalización del flamenco (su autonomía artística y su reconocimiento social) produjo sus herejías, como cualquier ortodoxia, las que encarnaron artistas como Marchena, Valderrama y, sobre todo, Manolo Caracol.

La principal aportación de este capítulo es analizar los elementos recurrentes de la mitología gitanista del mairenismo (razón incorpórea, pureza, hermetismo, etc.) bajo la luz de moldes analíticos bien asentados en la sociología de la cultura y de las manifestaciones artísticas ("modos de relación" de Claramonte; "usos tácticos" de Certeau; "infrapolíticas" de Scott o "habitus" de Bourdieu, entre otros). ${ }^{2}$ En cierto sentido, esta teorización sobre patrimonialidad pone el foco en el inquietante juego de fuerzas al que se ve sometido el artista: por una parte está en deuda con una trama cultural (simbólica, social y material) que le servirá de aval en el campo artístico: es su herencia; pero, al mismo tiempo, pugnará por una autonomía relativa, por manifestar su individualidad, tan cara a la noción y práctica del arte moderno. El concepto de tradición -que sin embargo no es problematizado por Aix suficientemente- es muy adecuado para subrayar esta ambivalencia. Aúna, por una parte, lo que, desde el pasado, es transmitido, seleccionado e interpretado en el presente, el factor colectivo y objetivo; simultáneamente, lo que es incorporado en el momento mismo de esa ejecución, por lo tanto transformado en el presente, el factor subjetivo, interno y más dinámico. De este modo, la invocación a la tradición no tiene que ser necesariamente un posicionamiento inmovilista, a no ser que la intención y el significado de los agentes sociales así lo definan. Como reconoce el autor, ha sido el crítico de flamenco Juan Vergillos quien más se ha aproximado a una lectura del flamenco como un par tradición/innovación.

El capítulo cuarto nos sitúa en la relación que el campo artístico del flamenco mantiene con los campos del poder en Andalucía. Para ello, y siguiendo de nuevo a Bourdieu, aborda el estudio del flamenco bajo una ciencia de las obras a través de tres operaciones fundamentales: (i) el estudio de las relaciones intercampo (planes de promoción institucional del flamenco, como el Plan Estratégico Sevilla 2000, Flamenco Porvenir, la candidatura del flamenco como Patrimonio Inmaterial de la Humanidad, la edición del disco Flamenco por Andalucía, España y la Humanidad); (ii) el análisis de la estructura

2. Del mismo modo que podría haber aplicado el de "culturas del trabajo" de los artistas flamencos, si bien este concepto pertenece al acervo teórico-metodológico de autores que entienden el flamenco, sobre todo, a través de su dimensión cultural (Moreno o Cruces). 
interna del campo artístico (relaciones intracampo); (iii) y el análisis de la génesis de los habitus de los ocupantes de estas posiciones. Tras poner el acento en las dinámicas e implicaciones del proceso de legitimación, institucionalización e instrumentalización del flamenco, que se traduce en una dependencia de los recursos públicos, concluye que se ha producido un paso desde la revalorización a la sobrevaloración institucional del flamenco, poniendo en peligro los logros de autonomía que el flamenco adquirió como campo durante su etapa fundacional y facilitando su decadencia como campo artístico (p. 316). Paradójicamente, una sentencia que el mismo autor critica a otros que la vienen anunciando desde el siglo XIX, como consecuencia de la mercantilización del flamenco.

El análisis de las políticas culturales e iniciativas institucionales va acompañado del estudio de algunos de los cambios más significativos que se observan en la evolución artística del espectáculo flamenco en el ámbito socio-profesional y en la presentación escénica, transformación que el autor sitúa en el paso de los festivales de verano a los grandes festivales globales: el baile se instala de nuevo en el teatro, frente al predominio que el cante tenía sobre este en el modelo anterior; el espontaneísmo y el voluntarismo dan paso al profesionalismo, perfeccionamiento y sofisticación de la puesta en escena; se asiste a una concentración de los recursos, contextos de distribución e instancias críticas de legitimación cultural en las llamadas ciudades globales, que tiene como revés la periferización del territorio cultural flamenco.

A la luz de sus reflexiones sobre la apertura estética actual se detiene, dada su relevancia, en el caso particular de la Bienal de Flamenco de Sevilla, para a partir de ahí tratar determinadas dinámicas como la proyección hacia los festivales globales, la deriva de lo que el autor denomina el citymarketing o la tendencia al estreno. La Bienal de Flamenco de Sevilla y la disyuntiva tradición/innovación, ya comentada, le permite adentrarse en la segunda operación que interesa al autor, el estudio de las relaciones intracampo -aunque en un nivel de profundidad menor que el dedicado a las relaciones intercampo, destilando una perspectiva netamente estructuralista-. Su aproximación a las posiciones sociales y las tomas de posición de los agentes, luchas y relaciones conflictuales, distribución de capitales, criterios de definición del valor artístico o el papel de los públicos y la afición ayudan a explicar cómo se configura la estructura interna del campo. La tercera de la operaciones abordadas, la génesis de las disposiciones y estrategias de los agentes recibe, también, un tratamiento desigual, y sin embargo en ella se tocan aspectos de gran interés para ahondar en lógicas, prácticas y transformaciones internas de plena actualidad, como el nuevo panorama de formación profesional, las posiciones en el campo en relación con la edad, la trayectoria de los artistas emergentes y la problemática de la precariedad y las alternativas laborales. La mirada sobre Israel Galván es la vía que emplea el autor para ilustrar algunos de los aspectos relativos a estas dos últimas operaciones. 


\section{Apuntes metodológicos}

Flamenco y poder pretende desarrollar una sociología crítica, cualitativa y reflexiva, si bien se resiste a abandonar el modelo positivo -al pretender reflejar la "realidad" del campo artístico del flamenco a partir de la propia posición. Es decir, a veces resulta difícil deslindar qué proyecta el autor desde su propio planteamiento teórico -que no puede dejar de ser ideológico- respecto de qué descubre una vez que se inserta en el campo social del flamenco. En un sentido similar, Nathalie Heinich (2001) cuestiona a la sociología del arte el énfasis en hacer prevalecer sus propias categorías de análisis sobre los discursos, los valores y las prácticas de los actores en un determinado campo artístico, sin haberse detenido suficientemente en la observación, descripción y análisis de la construcción de esas prácticas y valores, a partir de una más sólida fundamentación empírica. Esto es, reclama un ejercicio intelectual que se base menos en la validación/ invalidación de las prácticas y categorías de pensamiento de los actores de un campo por parte del sociólogo, y más en comprender cómo esos actores las construyen, las justifican y las emplean en sus discursos y en sus actos.

A veces puede resultar desconcertante la desigualdad en la extensión de los apartados, la ilación de los mismos, así como la combinación de un discurso teórico más alambicado con relatos sobre acontecimientos vividos en los que Aix deja ver demasiado descarnadamente su posición sin que ésta esté siempre teórica ni empíricamente justificada ${ }^{3}$. El autor se esfuerza en explicar técnicas etnográficas -delas que sí podríamos reprochar la inadecuada asimilación entre las ruedas de prensa y los grupos de discusión- y en definirse dentro del campo, con una reflexión crítica de la propia posición. El uso de fuentes secundarias predomina en el capítulo tercero, de corte histórico, mientras que en el capítulo cuarto se deja ver un arduo trabajo de campo basado en observación participante, análisis de prensa y documental. Ello no obstante, se echa de menos en el relato una mayor presencia de las voces de los agentes, resultado de las entrevistas cualitativas. Es un acierto el uso de la etnografía para los propósitos del libro, pues permite el análisis de las agencias de distintos actores del campo, encima y al margen de los escenarios, así como permite preguntarse por la recepción de los discursos (capital simbólico) de los agentes entre el público. Pero lo cierto es que el lector se queda con ganas de tener un mayor acceso a las perspectivas de los actores.

\section{Reflexión final}

Parafraseando, y jugando, con un inteligente juego de palabras acuñado por Luis Lavaur (1976) [El flamenco no es un arte popular, sino popularizado; no es un arte gitano, sino agitanado], una tesis central de Flamenco y Poder es que el flamenco no es un arte popular, sino populista, en el sentido de manifestación instrumentalizada, en

3. Como ocurre con su referencia al Congreso de Antonio Mairena celebrado en Sevilla en 2009 (p. 303 y ss.). 
nombre del pueblo, por agentes políticos, con el auxilio, voluntario o no, de intelectuales, profesionales, aficionados y críticos. No es ocioso recordar que La teoría romántica del flamenco de Lavaur supuso un torpedo en la línea de flotación del mairenismo como ideología legitimadora del flamenco, abriendo la senda en la que se inscribe el trabajo de Steingress, y a la que se acoge Francisco Aix. Es desde esta posición teórica desde la que denuncia la sobrevaloración institucional que el flamenco ha logrado con el gobierno autonómico, pues los custodios institucionales de este campo artístico, especialmente los representantes políticos, lo aherrojan en un populismo que impide el desarrollo de inercias estéticamente innovadoras, una denuncia que es compartida por un buen número de artistas (especialmente los que quedan excluidos de la centralidad del campo artístico), e incluso por algunos autores, como Moreno o Mandly, cuyas lecturas del fenómeno flamenco parecen tan distantes de la del propio autor.

Nos encontramos, en suma, ante una obra que consolida una de las líneas de la sociología flamenca reciente (el flamenco como género artístico), pero que abre otras nuevas, lo que calibramos como un mérito del libro. Así, si ambas dimensiones del flamenco, la etnicitaria/cultural y la artística, han coexistido históricamente, estableciéndose una intensa influencia mutua entre ambos planos, queda pendiente un estudio que focalice precisamente el trasiego de prácticas, reglas y valores de una a otra dimensión, sin entender este tránsito necesariamente como estrategias de instrumentalización política del flamenco. De hecho, la relación entre flamenco y poder no tiene por qué limitarse a las dinámicas de manipulación referidas. Inversamente, el flamenco podría devenir en instrumento de insurgencia y resistencia política desde la subalternidad. Siendo Francisco Aix protagonista y conocedor directo de distintas formas de uso del flamenco como herramienta performativa insurgente, podría abordar en un futuro esta menos transitada senda de la sociología del flamenco, pues cuenta con la formación y aún con el capital social para ello.

\section{REFERENCIAS BIBLIOGRÁFICAS.}

Ariño, Antonio (2002) "La patrimonialización de la cultura y sus paradojas en la sociedad del riesgo". En García Blanco, JM., Navarro Susaeta, P. (eds.), ¿Más allá de la Modernidad? Las dimensiones de la información, la comunicación y sus nuevas tecnologías. Madrid: Centro de Investigaciones Sociológicas, pp. 329-354.

Bourdieu, Pierre (1995) Las reglas del arte. Génesis y estructura del campo literario. Barcelona: Anagrama.

Cruces Roldán, Cristina (ed.), (1996) El flamenco: identidades sociales, ritual y patrimonio cultural. Jerez: Centro Andaluz de Flamenco. 
Cruces Roldán, Cristina. (2001) Flamenco y Patrimonio. Sevilla: Bienal de Flamenco de Sevilla.

Cruces Roldán, C. (2002) Más allá de la música. Sevilla: Signatura Ediciones.

Cruces Roldán, C. (2005) Más allá de la música II. Sevilla: Signatura Ediciones.

Heinich, Nathalie (2001) Lo que el arte aporta a la sociología. México DF: Sello Bermejo.

Lavaur, Luis (1976) Teoría romántica del cante flamenco. Madrid: Editorial Nacional

Mandly, A (2010) Los Caminos del Flamenco. Etnografía, Cultura y Comunicación en Andalucía. Sevilla: Signatura Ediciones.

Méndez, Lourdes (2009) Antropología del campo artístico. Del arte primitivo [...] al contemporáneo. Madrid, Síntesis.

Moreno Navarro, I. (1996) "El flamenco en la cultura andaluza”. En Cruces Roldán (ed.), El flamenco: identidades sociales, ritual y patrimonio cultural. Jerez: Centro Andaluz de Flamenco, pp. 15-33.

Steingress, Gerard (1993) Sociología del cante flamenco. Jerez de la Frontera: Centro Andaluz de Flamenco.

Steingress, Gerard (1996): "Ambiente flamenco y bohemia andaluza. Unos apuntes sobre el origen post-romántico del género gitano-andaluz". En Cruces Roldán, C. (Ed.), El flamenco: identidades sociales, ritual y patrimonio cultural. Jerez: Centro Andaluz de Flamenco, pp. 83-110.

Tatarkiewicz, Wladyslaw (1988): Historia de seis ideas. Arte, belleza, forma, creatividad, mímesis, experiencia estética. Madrid: Tecnos. 